\title{
Noninfectious Diarrhea in HIV Seropositive Individuals: a Review of Prevalence Rates, Etiology, and Management in the Era of Combination Antiretroviral Therapy
}

Patrick G. Clay • Rustin D. Crutchley

To view enhanced content go to www.infectiousdiseases-open.com

Received: August 13, 2014 / Published online: November 12, 2014

(C) The Author(s) 2014. This article is published with open access at Springerlink.com

\section{ABSTRACT}

Introduction: Diarrhea poses a substantial burden for patients with human immunodeficiency virus (HIV), negatively impacting quality-of-life (QoL) and adherence to antiretroviral therapy. During the combination antiretroviral therapy (cART) era, as incidence of opportunistic infection as a cause of diarrhea decreased, incidence of noninfectious diarrhea (including diarrhea as an adverse event [AE] of cART and HIV enteropathy) increased proportionately. A literature search was conducted for information on prevalence, etiology, and treatment options for noninfectious diarrhea in patients with HIV.

Electronic supplementary material The online version of this article (doi:10.1007/s40121-014-0047-5) contains supplementary material, which is available to authorized users.

P. G. Clay $(\bowtie)$

University of North Texas System College of

Pharmacy, 3500 Camp Bowie Boulevard, RES-340E,

Fort Worth, TX 76107, USA

e-mail: Patrick.Clay@unthsc.edu

R. D. Crutchley

Department of Clinical Science and Administration in the University of Houston College of Pharmacy,

Houston, TX, USA
Results: For marketed antiretroviral therapies, up to $28 \%$ of patients live with $>4$ loose or watery stools per day. The US Food and Drug Administration (FDA) does not require pharmaceutical manufacturers to include, within approved prescribing information, prevalence rates for all grades of diarrhea. Traditionally, noninfectious diarrhea management focused on avoiding use of diarrhea-associated cART; symptom management (nonpharmacologic and/or pharmacologic); and, as a last resort, changing cART. Examining the evidence upon which this approach is based reveals that most strategies rely upon anecdotal information and case reports. This review summarizes the literature and updates clinicians on the most recent options for management of noninfectious diarrhea in patients with HIV.

Conclusion: Diarrhea in patients with HIV is a significant unmet clinical need that contributes to worsening QoL and complicates medical management. Approaching management using a stepwise method of nonpharmacologic (diet), nonprescription (over-the-counter) and, finally, prescription agent changes (modification of cART or addition of an evidence-based 
antidiarrheal) appears reasonable, despite a lack of clear scientific evidence to support the initial two steps of this approach. If diet modifications, including psyllium and fiber introduction, fail to resolve noninfectious diarrhea in patients with HIV, loperamide followed by crofelemer should be considered. Clinicians are encouraged to review the most recent literature, not rely upon prescribing information. Continued vigilance by HIV providers to the presence of gastrointestinal AEs, even in patients taking the most recently approved antiretroviral agents, is warranted. Additional research is justified in identifying the etiology and management of HIV-associated diarrhea in patients on successful cART regimens.

Keywords: Antiretroviral therapy; Crofelemer; Diarrhea; Etiology; HIV enteropathy; Loose stools; Management; Prevalence; Loperamide

\section{INTRODUCTION}

Advances in the treatment of human immunodeficiency virus (HIV) and acquired immunodeficiency syndrome (AIDS) in the past decade have changed what once was considered a terminal illness into a manageable chronic disease [1]. Since the introduction of combination antiretroviral therapy (cART) in the mid-1990s, the life expectancy of individuals with HIV has increased dramatically [2-4]. As a result, the estimated number of individuals aged $\geq 13$ years living with AIDS in the United States has more than doubled between 1996 and 2008 (219,318 vs. 479,161 , respectively) [2]. Further, it is estimated that more than $50 \%$ of patients with HIV will be over the age of 50 years by 2015 [5]. Given the longevity achieved with current therapeutic strategies and the increased time patients are on these treatments because of improved efficacy, enhancing the quality of life (QoL) of patients with HIV by reducing symptoms associated with HIV and its treatment has become an important management goal.

Diarrhea remains an important clinical concern and is associated with reduced QoL in individuals with HIV during cART [6-8]. For example, diarrhea contributes to nonadherence or justification for switching from a currently successful cART regimen [7,9]. While the rate of opportunistic infections as a cause of diarrhea has decreased in the era of cART, there has simultaneously been a proportionate increase in the incidence of noninfectious diarrhea, often linked to use of antiretroviral therapy [7, 10]. Protease inhibitors (PI) and other antiretroviral medications may alter the function of the intestinal epithelial barrier and lead to secretory diarrhea, leaky-flux diarrhea, or both $[11,12]$. Detrimental effects of HIV infection itself on the gastrointestinal tract (i.e., HIV enteropathy) constitute another noninfectious cause of diarrhea [13]. In this review, prevalence, etiology, and current nonpharmacologic and pharmacologic management of noninfectious diarrhea in patients with HIV are described.

\section{METHODS}

A search of PubMed was conducted for general information on prevalence, etiology, and treatment options for noninfectious diarrhea in patients with HIV. For treatment updates, PubMed was searched using keywords ("HIV" AND "diarrhea") OR "HIV enteropathy" OR ("antiretroviral" AND "diarrhea") and limited to clinical studies published in English from January 1995 to July 2014. To identify 
treatment updates on over-the-counter and complementary medicines, the Natural Medicine Comprehensive Database was searched using the keywords "HIV and diarrhea". Bibliographies from included articles were manually reviewed for additional relevant studies. Because of the degree of variance in how diarrhea incidence is reported in clinical trials, the most recent US Food and Drug Administration (FDA)-approved prescribing information was also reviewed for each antiretroviral drug. Additional treatments in development were identified by searching ClinicalTrials.gov.

This article is a review and does not contain any new studies with human or animal subjects performed by any of the authors.

\section{Prevalence of Noninfectious Diarrhea}

Using observational data to determine prevalence rates is sometimes deemed less valuable than using data from randomized controlled studies. However, evidence exists that with respect to true rates of adverse events (AEs), like noninfectious diarrhea, data from observational studies and randomized controlled trials are comparable [14]. In order to facilitate reading from either viewpoint, this review has separated observational data from data that have been obtained from controlled clinical studies.

\section{Observational Studies}

Before the widespread use of cART, up to $70 \%$ of patients with HIV reported the development of diarrhea [10, 15]. From 1995 to 1997 [7, 10, 16$18]$, as the use of cART increased to $>80 \%$ of patients [19], overall rates of diarrhea decreased but, importantly, the causality changed from infectious to noninfectious [10]. This is reflected in Table 1 [7, 10, 16-18]. The incidence of opportunistic infections causing diarrhea decreased from $53 \%$ to $13 \%$, whereas noninfectious causes of diarrhea increased from $32 \%$ to $70 \%$ [10]. The predominant cause of noninfectious cases was medicationassociated diarrhea, which increased from 0\% in 1995 to $45 \%$ in 1997 . The medications most commonly used by patients during this period were PIs (e.g., nelfinavir, ritonavir, saquinavir, and indinavir) and a nucleoside reversetranscriptase inhibitor (NRTI; didanosine) [10].

Today, despite improvement in gastrointestinal tolerability of the newer agents (including the one most recently approved, dolutegravir) [20], diarrhea remains a common condition in patients with HIV, including those experiencing virologic and immunologic success [7]. Two cohort studies found diarrhea rates ranging from 39\% [16] to $60 \%$ [17] and, in one instance, the incidence of diarrhea in a population of people with HIV was quadruple the rate reported for a non-HIV population [7]. Considering the influence of the class of cART, patients receiving PIs had a higher incidence of diarrhea compared with those on PI-sparing regimens (38\% vs. $17 \%$, respectively) [7]. The impact on patients' lives is best reflected by a 2008 cross-sectional survey of patients with HIV taking cART $(n=953)$, in which diarrhea was ranked second $(63.0 \%)$ only to fatigue $(70.7 \%)$ as the most common medication-related AE [18].

\section{Randomized Clinical Studies as Cited in Individual cART Component Package Inserts}

The FDA does not standardize or mandate the specific manner in which pharmaceutical manufacturers should report diarrhea within the approved prescribing information (also referred to as package inserts). Table 2 outlines the various ways in which these data are 
Table 1 Incidence of diarrhea in observational studies

\begin{tabular}{|c|c|c|c|}
\hline Study & Study description (year) & $N$ & Key results \\
\hline Call et al. $[10]$ & $\begin{array}{l}\text { Retrospective study of patients with HIV } \\
\text { receiving primary care at outpatient HIV } \\
\text { clinic (1995-1997) }\end{array}$ & $\begin{array}{r}436 \text { in } \\
1995 \\
582 \text { in } \\
1996 \\
739 \text { in } \\
1997\end{array}$ & $\begin{array}{l}\text { Patients with opportunistic infection etiologies } \\
\text { decreased from } 53 \% \text { to } 13 \% \text {; patients with } \\
\text { noninfectious diarrhea increased from } 32 \% \text { to } \\
70 \%{ }^{a}\end{array}$ \\
\hline Knox et al. [16] & $\begin{array}{l}\text { Nutrition for Healthy Living study in } \\
\text { inpatient, outpatient settings } \\
(1996-1997)\end{array}$ & 671 & $39 \%$ of patients reported diarrhea ${ }^{b}$ \\
\hline \multirow[t]{2}{*}{$\begin{array}{l}\text { Zingmond et al. } \\
{[17]}\end{array}$} & $\begin{array}{l}2 \text { cross-sectional cohorts: } \\
\text { HIV Cost and Service Utilization Study } \\
(1996-1997)\end{array}$ & $\begin{array}{l}3,745 \\
2,864\end{array}$ & $53 \%$ of 3,745 patients reported diarrhea ${ }^{c}$ \\
\hline & $\begin{array}{l}\text { Veterans Aging Cohort } 3 \text { Site Study } \\
(1999-2000)\end{array}$ & 881 & \\
\hline Siddiqui et al. [7] & $\begin{array}{l}\text { Prospective study recruiting patients in } \\
\text { infectious disease and primary care } \\
\text { clinics }(2002-2003)\end{array}$ & 416 & $\begin{array}{l}28.2 \% \text { of patients with HIV vs. } 7.1 \% \text { in HIV- } \\
\text { seronegative controls }(p<0.001) \text { reported } \\
\text { diarrhea }^{d}\end{array}$ \\
\hline $\begin{array}{l}\text { daCosta } \\
\text { DiBonaventura } \\
\text { et al. }[18]\end{array}$ & $\begin{array}{l}\text { Cross-sectional self-reported survey of } \\
\text { patients with HIV receiving cART } \\
(2008)\end{array}$ & 953 & $\begin{array}{l}63 \% \text { of patients reported diarrhea }{ }^{c} \text { as cART- } \\
\text { related adverse effect }\end{array}$ \\
\hline
\end{tabular}

cART combination antiretroviral therapy, $H I V$ human immunodeficiency virus

a Diarrhea defined as $>3$ bowel movements per day for $>2$ weeks

b Diarrhea defined as (1) interfering with normal activity in the past month in a self-reported range of "very little or slightly" to "extremely" (severe diarrhea of $\geq 6$ watery bowel movements per day), or ( 2$) \geq 3$ loose or watery stools per day in the past month

c Patient-reported symptom

d Diarrhea defined as $\geq 3$ bowel movements per day in the past 7 days

presented (by grade, causality, treatment discontinuation, etc.). Package inserts infrequently disseminate incidence rates for all severities of diarrhea. Instead, most only report rates using terms such as "moderate" and "severe" diarrhea, with some including the actual percentages of patients meeting a particular definition (e.g., $>4$ loose or watery bowel movements per day). In one example, prescribing information for the non-nucleoside reverse-transcriptase inhibitor (NNRTI) class only reported grade 2 or higher severity of diarrhea using the Division of AIDS grading scale (DIADS) definition [21], which translated to increase from baseline of 4-6 loose or watery bowel movements per day, poorly reflecting the true incidence of loose stools in persons being prescribed these agents today.

Up to $28 \%$ of patients taking today's commonly used antiretroviral therapies deal with $>4$ loose or watery stools per day (see Table 2) [22]. According to prescribing 
Table 2 Incidence of diarrhea in patients with HIV for currently marketed antiretroviral therapies based on prescribing information [22]

\begin{tabular}{lll}
\hline Drug name & Diarrhea rate, \% & $\begin{array}{l}\text { Diarrhea severity } \\
\text { or grade }^{\mathrm{a}}\end{array}$ \\
\hline
\end{tabular}

Multiclass combination products

Efavirenz, emtricitabine, and tenofovir disoproxil fumarate

Emtricitabine, rilpivirine, and tenofovir disoproxil fumarate

Elvitegravir, cobicistat, emtricitabine, and tenofovir disoproxil fumarate

\section{NRTIs}

Zidovudine

Abacavir and lamivudine combination product

Abacavir

Abacavir, zidovudine, and lamivudine combination product

Tenofovir, disoproxil fumarate, and emtricitabine combination product

Emtricitabine

Tenofovir

Lamivudine

Lamivudine and zidovudine combination product

Didanosine

Stavudine

Enteric-coated didanosine

NNRTIs

Etravirine

Immediate release; nevirapine

Rilpivirine

Delavirdine

Efavirenz

Extended release; nevirapine

Protease inhibitors

$\begin{array}{lll}\text { Atazanavir } & 1-3^{\mathrm{i}} & \text { Moderate to severe } \\ \text { Indinavir } & 3 & \text { Moderate to severe } \\ \text { Saquinavir } & 8^{\mathrm{j}} & \text { Grades 2-4 } \\ \text { Darunavir } & 9^{\mathrm{h}, \mathrm{j}} & \text { Grade } \geq 2 \\ \text { Fosamprenavir } & 13^{\mathrm{ij}} & \text { Moderate to severe }\end{array}$

Grades 2-4

All grades

All grades

$<5$

$5^{\mathrm{b}}$

$7^{\mathrm{c}}$

7

$9^{\mathrm{b}}$

$\geq 10$

$11^{\mathrm{c}}$

18

18

19

50

$57^{\mathrm{d}}$

All grades

Grades 2-4

Grades 2-4

Grades 2-4

Grades 2-4

All grades

Grades 2-4

All grades

All grades

All grades

All grades

All grades

Grades 2-4

$<1-2^{\mathrm{e}}$

$<2^{\mathrm{e}}$

$2^{\mathrm{f}}$

$3^{\mathrm{g}}$

$4^{\mathrm{h}}$

Grade $\geq 2$

Grades 2-4

Moderate to severe

Grades 2-4

Moderate to severe

to severe

Moderate to severe 
Table 2 continued

\begin{tabular}{|c|c|c|}
\hline Drug name & Diarrhea rate, \% & $\begin{array}{l}\text { Diarrhea severity } \\
\text { or grade }\end{array}$ \\
\hline Tipranavir & $15^{j}$ & $\begin{array}{l}\text { Moderate to severe } \\
\text { (Grades 2-4) }\end{array}$ \\
\hline Ritonavir & $15-23$ & Moderate to severe \\
\hline Lopinavir and ritonavir combination product & $15-28^{\mathrm{i}}$ & Moderate to severe \\
\hline Nelfinavir & $20^{\mathrm{k}}$ & Moderate to severe \\
\hline \multicolumn{3}{|l|}{ Fusion inhibitors } \\
\hline Enfuvirtide & $32^{\mathrm{e}}$ & All grades \\
\hline \multicolumn{3}{|l|}{ Entry inhibitors } \\
\hline Maraviroc & $\leq 1^{\mathrm{e}}$ & All grades \\
\hline \multicolumn{3}{|l|}{ Integrase inhibitors } \\
\hline Dolutegravir & $\leq 1^{\mathrm{i}}$ & Moderate to severe \\
\hline Raltegravir & $\leq 1^{\mathrm{h}}$ & Moderate to severe \\
\hline
\end{tabular}

$A E$ adverse event, NRTI nucleoside reverse-transcriptase inhibitors, NNRTI nonnucleoside reverse-transcriptase inhibitors

${ }^{a}$ Grade 1 (mild): temporary episodes of unformed stools or increase from baseline of $\geq 3$ stools during a 24-h period. Grade

2 (moderate): persistent episodes of unformed to watery stools or increase from baseline of 4-6 stools during a 24-h period.

Grade 3 (severe): bloody diarrhea or increase from baseline of $\geq 7$ stools during a 24-h period or intravenous fluid supplementation required. Grade 4 (potentially life-threatening): potentially fatal [23]

b With efavirenz

c With lamivudine and efavirenz

d With nelfinavir and stavudine

e With background regimen

${ }^{f}$ With zidovudine

g With zidovudine and lamivudine

$h$ With tenofovir and emtricitabine

i With NRTI backbone

j Ritonavir-boosted regimen

$\mathrm{k}$ With stavudine and lamivudine

information, PIs demonstrate the highest average prevalence of diarrhea (13.6\%) compared with NRTIs (10.0\%, excluding didanosine and stavudine, which are not used commonly in clinical practice today because of greater associated toxicities), while the average rate of diarrhea with NNRTIs is much lower (2.2\%). Taken individually, package inserts for the integrase inhibitors (raltegravir and dolutegravir) and the $\mathrm{C}-\mathrm{C}$ motif receptor 5 (CCR5) antagonists (maraviroc) report some of the lowest incidences of diarrhea $(\leq 1 \%$ each class). These figures are in contrast with a comprehensive report using consistent DIADSbased reporting of gastrointestinal AEs [21], in which up to $19 \%$ of patients treated with cART reported grades 2 through 4 diarrhea (i.e., moderate to potentially life-threatening diarrhea) [23].

Protease Inhibitors Among the antiretroviral therapy classes, diarrhea is most commonly 
associated with PIs [24]. More recently approved agents in this class have a reportedly lower incidence of diarrhea than older agents (Table 2) [24-27]. The primary use of ritonavir is as a pharmacokinetic booster for other PIs (100 mg once daily with atazanavir or darunavir [in treatment-naïve patients with no documented darunavir-resistant HIV mutations]; $100 \mathrm{mg}$ twice daily with lopinavir, fosamprenavir, or darunavir [in treatmentexperienced patients with darunavir-resistant HIV mutations]; or $200 \mathrm{mg}$ twice daily with tipranavir). Partly due to the frequency of gastrointestinal AEs, ritonavir is rarely used today as the sole PI (600 mg twice daily) [23]. All ritonavir-boosted PIs cause some degree of diarrhea, but data from clinical studies reveal differences in rates of diarrhea according to the PI being used [28]. Lopinavir/ritonavir (10-11\%) and fosamprenavir/ritonavir (13\%) cause higher rates of drug-related moderate to severe diarrhea in treatment-naïve patients compared with atazanavir/ritonavir (2\%) and darunavir/ ritonavir (4\%) therapies [25-27].

\section{Nucleoside/Nucleotide Reverse-Transcriptase}

Inhibitors It is challenging for clinicians to understand how the NRTIs commonly used today contribute to diarrhea rates in cARTtreated patients, as clinical studies with NRTIs report gastrointestinal AEs inconsistently [29, 30]. In the HIV Study With Epzicom and Truvada ([EPZ104057; ClinicalTrials.gov identifier NCT00244712]) study of abacavir/ lamivudine versus tenofovir disoproxil fumarate/emtricitabine $\quad(N=688), \quad$ the incidence of grades 2-4 drug-related diarrhea was similar in the tenofovir/emtricitabine and abacavir/lamivudine treatment groups (19\% for both arms) in treatment-naïve patients [29]. However, these patients also received concomitant lopinavir/ritonavir, which is individually reported to generate grade 2 or higher diarrhea in $10-11 \%$ of patients $[25,26]$. Clinicians must interpret at the patient level if the additional $8-9 \%$ incidence of diarrhea is attributable to the NRTI backbone or other causes. A review of 13 studies evaluating PIsparing NRTI plus NNRTI cART regimens (included NRTIs were tenofovir/emtricitabine, tenofovir/lamivudine, or zidovudine/ lamivudine) in antiretroviral therapy-naïve patients $(N=3649)$ reported that up to $38 \%$ (range 0-38\%) of patients experienced diarrhea (all definitions of diarrhea were considered) [30].

\section{Non-Nucleoside Reverse-Transcriptase}

Inhibitors Reporting of AEs in clinical trials with NNRTIs (first-generation [efavirenz and nevirapine] and second-generation [etravirine and rilpivirine]) predominantly limited diarrhea severity data to grades 3 or 4 (or the patient having bloody diarrhea, an increase from baseline of $\geq 7$ stools during a 24-h period, or a need for intravenous fluid supplementation) [23]. This approach appears to have resulted in gastrointestinal AEs to be reported as rarely occurring. In the AIDS Clinical Trials Group Study A5142 trial (ClinicalTrials.gov identifier: NCT00050895) of treatment-naïve patients, the percentage of patients reporting grade 3 or 4 diarrhea was $<1 \%$ in the treatment arm receiving efavirenz plus 2 NRTIs and 3\% in the lopinavir/ritonavir plus efavirenz arm [31]. The apparently lower rate of diarrhea reported for lopinavir/ ritonavir may be an artifact of authors reporting only grade 3 or worse diarrhea instead of grade 2 or worse and not reflective of all grades of diarrhea experienced in the population studied. This supports the theory that clinicians are challenged to know the true incidence of 
clinically meaningful diarrhea in patients due to the lack of standardized reporting and less inclusive FDA labeling requirements.

Notably distinct is the reporting of all grades of diarrhea for etravirine in clinical trials. The rate of all-grade diarrhea in adults was lower for etravirine versus placebo ( $18 \%$ vs. $24 \%$ ) in a pooled 48-week analysis of the Demonstrate Undetectable viral load in patients Experienced with ARV Therapy studies (DUET-1 [TMC125C206] and DUET-2 [TMC125-C216]; ClinicalTrials.gov identifiers NCT00254046 and NCT00255099, respectively) [32]. The lower incidence versus placebo, although substantial, may help explain why no data on the incidence of diarrhea in adults are reported in the FDA-approved label.

Integrase Inhibitors In clinical trials, integrase inhibitors (raltegravir, elvitegravir, dolutegravir) demonstrated incidences of moderate to severe diarrhea similar to those observed with placebo. The raltegravir studies (Blocking Integrase in Treatment Experienced Patients with a Novel Compound against HIV BENCHMRCK-1 [MK0518] and BENCHMRCK-2 [MK-0519]; ClinicalTrials.gov identifiers NCT00293267 and NCT00293254, respectively) of patients with treatment-resistant HIV infection reported an $\sim 4 \%$ incidence of moderate to severe diarrhea in both the raltegravir and placebo groups [33]. This rate is notably distinct from the incidence of diarrhea of all grades, which was reported in the publication supplement to be $18.4 \%$ in the raltegravir group and $21.1 \%$ in the placebo group [33]. From a clinician standpoint, while it is valuable to know the difference in rates between arms of the trial, having a better idea of the true incidence that is likely to occur in patients is also important.
Fixed-Dose Combination Antiretroviral Products Incidence of diarrhea with fixeddose combination, single-tablet regimens appears to be based on the side effect profiles of the component medications or, at times, a compilation of data from various studies involving the coformulated agents and the data used in FDA-approved package inserts. For instance, in a 48 -week phase 3 clinical trial of efavirenz/emtricitabine/tenofovir disoproxil fumarate, diarrhea of any grade was reported in $8 \%$ of patients [34]. However, in the prescribing information for this formulation, the incidence of moderate to severe diarrhea is reported as $9 \%$ (Table 2). The publication on the phase 3, 48-week, clinical trial of elvitegravir/cobicistat/ emtricitabine/tenofovir disoproxil fumarate reported an incidence of $22 \%$ for diarrhea of any grade [35]. However, the incidence of all grades of diarrhea in the approved labeling is only $12 \%$. This variance in incidence and severity reporting (12\% vs. $22 \%)$ reinforces the need for individual patients prescribed this combination agent to be assessed by their clinicians for the occurrence of loose stools.

As summarized earlier, clinicians are likely to face challenges when it comes to predicting the true rates of diarrhea occurring in persons taking CART, using only prescribing information. Accordingly, regardless of which combination of antiretroviral therapy is initiated, patients should be counseled to report loose, watery stools or diarrhea to enable their medical providers to determine whether the etiology of the patient's diarrhea is infectious (not covered in this review) or noninfectious.

\section{Etiology of Noninfectious Diarrhea}

The etiology of noninfectious diarrhea in patients with HIV is multifactorial and most 
likely includes (in decreasing order of influence) cART-associated diarrhea; the detrimental effects of HIV on the gastrointestinal tract (i.e., HIV enteropathy); and, infrequently, HIVassociated malignancies and pancreatitis [36].

\section{cART-Associated Diarrhea}

There are two primary theoretical mechanisms by which cART may cause diarrhea: damage to the intestinal epithelial barrier, which leads to "leaky-flux" diarrhea, and altered secretion of calcium-dependent chloride ions, which causes secretory diarrhea [11, 12, 37].

Intestinal barrier damage: In in vitro and in vivo studies, PIs induced endoplasmic reticulum stress and activated an unfolded protein response, leading to apoptosis of intestinal cells [38]. Two caveats to these data are (1) the concentrations of PIs used in these studies substantially exceeded those obtained in humans treated with the FDA-approved doses of PIs [6], and (2) no substantiation of the leakyflux model for cART-associated diarrhea in humans has been published.

Altered secretion of chloride ions: Secretory diarrhea is characterized by increased secretion of the chloride ion $\left(\mathrm{Cl}^{-}\right)$into the lumen, with subsequent sodium and water accompaniment [39]. Studies specifically evaluating PIs have demonstrated induction of secretory diarrhea via the potentiation of calcium-activated chloride conductance [37], but published human data supporting this concept are limited. A botanical agent that acts as a dual inhibitor of the cyclic adenosine monophosphate (cAMP)-stimulated cystic fibrosis transmembrane conductance regulator (CFTR) chloride channel and the calciumactivated chloride channel (CaCC) was shown to improve symptoms of diarrhea and decrease stool chloride concentrations in patients with HIV receiving cART $[40,41]$.

\section{HIV Enteropathy}

HIV enteropathy has been proposed to result from viral infection of gastrointestinal enterocytes, leading to a loss of the intestinal epithelial barrier function of the gastrointestinal mucosa [13, 42, 43]. This loss may manifest as villous atrophy, crypt hyperplasia, and villous blunting, leading to increased permeability [44]. Consequent activation of the gastrointestinal immune system, leading to inflammation, may further trigger barrier degradation. Finally, a welldescribed complication of HIV infection is HIV directly damaging the autonomic nerves of the intestine, leading to faster intestinal transit times [6].

\section{Implications of Noninfectious Diarrhea in Patients with HIV}

Diarrhea is known to complicate the management of patients with HIV by contributing to nonadherence or causing a need for modification of an otherwise virologically and immunologically successful regimen [7, 9]. Especially concerning is when patients independently decide to discontinue cART due to diarrhea. In a retrospective study analyzing first-line antiretroviral therapy discontinuation during a 22-year period $(1,861$ patient-years of follow up), gastrointestinal symptoms were the most common AEs associated with treatment discontinuation [9]. Adverse event management guidelines recommend careful evaluation of the symptoms and symptomatic management of AEs before switching antiretroviral therapy if AEs are mild to moderate in severity (particularly with diarrhea and nausea) [45]. As has been illustrated earlier in this review, providers are hampered in their ability to ascertain the true rates of mild to moderate 
diarrhea for cART agents in use today. This forces healthcare providers to potentially base their decisions on regimen component changes on less than optimal information [22]. Switching from one antiretroviral therapy to another is a feasible option, although patients who switch therapy are at an increased risk for reduced QoL [46]. Further, there are a limited number of times for which patients can switch therapies before recycling of antiretroviral agents occur [47]. Therefore, symptomatic management of mild to moderate AEs before switching from a successful antiretroviral regimen may be preferable.

\section{Management of Noninfectious Diarrhea}

As a general rule, management of medical conditions begins with lifestyle modification followed by alteration of the offending agent (if considered to be the etiology). When these methods fail, clinicians then resort to available "therapies." Agents selected should result from careful consideration of input from both the patient/caregiver and existing medical literature. Balancing these datasets, healthcare providers develop personal algorithms for management, especially when clear guidance is not available. HIV-associated diarrhea (in patients optimally on stable, successful ART) lacks standardized guidelines. Presented below is a compendium of options with varying degrees of available data and successful outcomes. Where possible, a suggested order of use in patients is provided.

Successful management of diarrhea in patients with HIV has a number of important potential benefits, including improving adherence to cART, enabling patients to maintain an otherwise successful regimen, improving nutritional status, stabilizing weight, and enhancing QoL [8, 48, 49].
Management of noninfectious diarrhea in patients with HIV receiving cART initially should include supportive nonpharmacologic treatments, followed by pharmacologic treatments, with cART modification as a secondary option. However, limited evidence exists for the use of nonpharmacologic and pharmacologic approaches [49-55]. This review focused on literature involving patients with HIV, as cART requires special considerations.

\section{Nonpharmacologic and Complementary Alternative Medicine Approaches}

Traditional nonpharmacologic agents for the symptomatic treatment of diarrhea in patients with HIV include dietary modifications, use of fiber, and administration or application of other supplements (see Table 3) [49, 51-55].

\section{Dietary Modification}

Unfortunately, for the evidence-driven clinician, scientific data supporting the effectiveness of dietary interventions for managing diarrhea in patients with HIV are sparse. A single clinical study of symptom management in HIVassociated diarrhea $(N=75)$ using normal foods (i.e., a low-fat, low insoluble fiber, lactose-free, high soluble fiber, and caffeine-free diet) versus a control group without dietary restrictions reported that after 24 weeks, dietary intervention was effective in reducing stool frequency $(p=0.0003)$ and improving stool consistency ( $p=0.0002)$ [51]. This approach is reasonable and should be encouraged. It is also potentially a beneficial lifestyle modification for patients with HIV, especially those with comorbid conditions of diabetes and obesity.

\section{Bulking Agents}

Classified by the FDA as food supplements, psyllium and oat bran are bulking agents. 
Table 3 Nonpharmacologic treatment for noninfectious diarrhea in patients with HIV [49, 51-55]

\begin{tabular}{|c|c|}
\hline Agent/intervention & Theorized mechanism of action \\
\hline Dietary intervention & Reduces caffeine, fat, and lactose intake, and increases soluble fiber consumption \\
\hline Psyllium & Increases fiber content and acts as a bulking agent \\
\hline Oat bran & Increases soluble fiber and acts as a bulking agent \\
\hline Probiotics & Modulate and normalize enteric flora \\
\hline $\begin{array}{l}\text { Bovine serum } \\
\text { immunoglobulin }\end{array}$ & Restores mucosal immunity and gastrointestinal function by reducing intestinal inflammation \\
\hline L-Glutamine & Energy source for cells, such as enterocytes; preserves gut mucosal integrity \\
\hline Curcumin & Anti-inflammatory agent \\
\hline $\begin{array}{l}\text { Acupuncture with } \\
\text { moxibustion }\end{array}$ & $\begin{array}{l}\text { Affects gastric motility, electrical activity, and secretion of gastric acid, pancreatic enzymes, and } \\
\text { bile by stimulating the points along the stomach or spleen channels }\end{array}$ \\
\hline
\end{tabular}

Although bulking agents are commonly employed to remedy diarrhea, historically they have shown limited utility in patients with HIV and diarrhea [49, 56, 57]. Data do not exist regarding use of bulking agents with today's commonly used antiretroviral agents. As an additive or adjunct therapy with dietary modifications, it is relatively inexpensive and there appears little harm to attempting this remedy, if agreeable to the patient.

\section{Probiotics}

Probiotics, often promoted to "enhance" the immunologic health of the gastrointestinal system [58, 59], have been studied in the treatment and prevention of diarrhea in patients with HIV. While the mechanism for the effects of probiotics on gastrointestinal immune health have been reported on in more detail elsewhere, the existing evidence for efficacy is conflicting [60]. In a randomized, crossover, placebo-controlled study in $17 \mathrm{HIV}$ infected patients with noninfectious diarrhea receiving antiretroviral therapy, the majority of whom (89\%) were receiving PIs (nelfinavir or ritonavir), therapy with the probiotic Lactobacillus rhamnosus GG for 2 weeks did not significantly affect the frequency or consistency of stools compared with placebo. However, in another randomized, double-blind, clinical study in patients with HIV not receiving antiretroviral therapy, probiotic yogurt containing mixed Lactobacilli strains resolved moderate diarrhea in all 12 patients within 2 days of treatment compared with 2 of 12 patients receiving nonprobiotic yogurt (i.e., control group) for 15 days [52, 61]. In a larger placebo-controlled, randomized study, 69 patients with HIV who were also naïve to antiretroviral therapy received treatment with Lactobacilli strains for 25 weeks but reported no significant modification of diarrheal symptoms compared with placebo [62]. At present, the use of probiotics for the treatment of noninfectious diarrhea in patients with HIV (especially those receiving cART) is not supported in the literature. However, the limited body of evidence does not reflect the wide diversity of probiotics available that have yet to be tested and may demonstrate verifiable efficacy in the future. This approach, similar to bulking agents, falls into the unscientifically grounded category of "unlikely to cause harm" and seems reasonable if favored by the patient. 


\section{Bovine Serum-Derived Immunoglobulin}

This agent is known to reduce inflammation of the intestine, restore mucosal immunity, and improve gastrointestinal function [63]. In a small, open-label, 8-week study in eight patients with HIV enteropathy, treatment with oral serum-derived bovine immunoglobulin reduced the median number of bowel movements per day $(5.8-2.0 ; p=0.008)$ and improved stool consistency, as scored on a scale of 1 (formed) to 6 (watery) $(5.3-3 ; p=0.008)$. A clinical trial of this agent in the treatment of patients with HIV-associated enteropathy is currently ongoing (ClinicalTrials.gov identifier: NCT01828593). This approach, addressing potentially two of the theoretical causes of HIV enteropathy, is scientifically sound. It shows promise, but definitive data are pending. Bovine serum-derived immunoglobulin is currently available as a medical food and is FDA approved for use in clinical dietary management of enteropathy, including HIV-associated forms [64]. Its place within the treatment decision algorithm should be known following completion of ongoing clinical trials.

\section{L-Glutamine}

In a randomized, placebo-controlled study of 25 patients with HIV and nelfinavir-induced diarrhea, supplementation with L-glutamine significantly reduced the severity of diarrhea. Based on a 5-point scale for assessment of bowel movements, with normal $=$ grade 0 and $>7$ stools/day $=$ grade 4 , the mean grade of diarrhea for patients taking L-glutamine was 0.76 , compared with a mean grade of diarrhea of 1.85 for patients who received placebo; $p<0.01[53,65]$. However, this approach has not been replicated by others, nor has it been evaluated using cART agents commonly prescribed today. Further data would be beneficial to interpret where L-glutamine fits into the treatment paradigm.

\section{Curcumin}

The beneficial effects of curcumin have been reported in the treatment of various disease states. The mechanism of action of curcumin in diarrhea is theorized to be related to its antiinflammatory properties (the reader is referred to Dulbecco and Savarino 2013 [66] for a comprehensive review of this agent and its potential medicinal properties). Curcumin is a naturally occurring hydrophobic phenol extracted from turmeric, which is best known as the primary spice in curry or the yellowing agent for some varieties of mustard. With respect to HIV-associated diarrhea, there is a single clinical study $(n=8)$ of patients treated with curcumin for a mean of 41 weeks (7/8 patients were receiving cART) [54]. In this case series of eight patients, curcumin therapy resulted in rapid (mean 13 days) and complete resolution of diarrhea and normalization of stool quality. No additional studies replicating these results with curcumin have been published. More standardized studies of the efficacy of curcumin as a therapeutic agent for diarrhea are needed before curcumin treatment can be recommended. Clinicians who wish to consider adding it to patients' diet as a dietary supplement are encouraged to first formally evaluate the balance necessary between other dietary elements and curcumin concentration to maintain the antidiarrheal effect of curcumin.

\section{Acupuncture}

The combination of acupuncture and moxibustion (application of heat generated by burning of an herbal mixture containing Artemisia) has been used to address the bowel patterns of patients with HIV; however, 
evidence for the effectiveness of this therapy is limited [55]. While many patients with HIV may receive acupuncture to alleviate symptoms, it would likely take a clinical research network-generated, peer-reviewed publication describing the antidiarrheal effect of acupuncture and moxibustion to fully inform practicing clinicians and patients alike on this particular use. Until sufficient data are available, this treatment modality cannot be recommended.

\section{Pharmacologic Options}

\section{Adsorbents}

Adsorbents such as bismuth subsalicylate and attapulgite act by adsorbing fluids and compounds to improve stool consistency (see Table 4) [67]. This approach for managing HIVassociated noninfectious diarrhea has been considered based on the high degree of success observed in some patients using adsorbents to treat travelers' diarrhea [68, 69]. However, the etiology and pathophysiology of travelers' diarrhea is distinct from that of HIV-associated noninfectious diarrhea, and the success of therapies for travelers' diarrhea has not transferred to the setting of HIV [70]. Clinical evidence for the use of these agents in controlling diarrhea in the HIV population is limited to one randomized, double-blind study that reported that attapulgite was no better than placebo for improving stool consistency or stool frequency in adults with HIV and diarrhea $[67,71]$. These agents cannot be recommended for use at this time for the treatment of HIVassociated noninfectious diarrhea.

\section{Antimotility Agents}

Oral antimotility agents such as loperamide and diphenoxylate/atropine are opioid derivatives, which increase fecal transit time by decreasing bowel activity and increasing tone in the large intestine. These actions, in turn, provide greater opportunity for the potentially leaky gastrointestinal tract to absorb fluid from the gut [67].

Loperamide The best evidence for chronic use of loperamide in patients with HIV comes from a study that included concomitant use of

Table 4 Pharmacologic treatment for noninfectious diarrhea in patients with HIV [50, 67, 73]

\begin{tabular}{|c|c|c|}
\hline Drug & Mechanism of action & $\begin{array}{l}\text { Adverse effects/drug-drug interactions } \\
\text { and additional information }\end{array}$ \\
\hline $\begin{array}{l}\text { Adsorbents (attapulgite and } \\
\text { bismuth subsalicylate) }\end{array}$ & Adsorbent & $\begin{array}{l}\text { Can cause constipation; no evidence of drug-drug } \\
\text { interactions with components of cART }\end{array}$ \\
\hline Loperamide & Antimotility agent & $\begin{array}{l}\text { Cramping, constipation; potential drug interaction } \\
\text { with numerous antiretroviral therapies; familiar to patients }\end{array}$ \\
\hline Diphenoxylate/atropine & Antimotility agent & Abuse potential \\
\hline Octreotide & Antisecretory agent & $\begin{array}{l}\text { Potentially serious hormonal and gastrointestinal } \\
\text { disturbances; injectable }\end{array}$ \\
\hline Crofelemer & Antisecretory agent & $\begin{array}{l}\text { Low rate of AEs; no clinically relevant drug-drug } \\
\text { interactions }\end{array}$ \\
\hline
\end{tabular}

$A E$ adverse event, $c A R T$ combination antiretroviral therapy 
antimicrobial treatment for intestinal infections, which does not allow for conclusions to be drawn on loperamide efficacy in the management of noninfectious diarrhea [72]. A retrospective study, published in abstract form in 1998 and cited in reviews, indicated that $32 \%$ of patients with HIV receiving nelfinavir responded to loperamide treatment for the management of diarrhea [49]. However, the use of loperamide, particularly for long-term management, may be limited by treatment-related AEs, including drug interactions with antiretroviral therapy [73, 74]. As a P-glycoprotein (P-gp) substrate unable to cross the blood-brain barrier (P-gp efflux pump prevents this translocation), loperamide generally only possesses peripheral opioid activity [75]. Although PIs (as P-gp inhibitors) could increase loperamide levels in the brain, potentially causing respiratory depression [76], formal evaluation of coadministration of loperamide with ritonavir-boosted tipranavir did not result in any clinically relevant central nervous system opioid effects [75]. Given the substantial clinical history of use of loperamide to treat diarrhea in patients with HIV, despite minimal supporting peer-reviewed evidence, loperamide represents a relatively safe and inexpensive first-line approach.

Diphenoxylate/Atropine Data on the use of diphenoxylate/atropine for the treatment of diarrhea in patients with HIV are sparse. There are anecdotal, non-peer-reviewed reports of low-dose (1-5 mg) diphenoxylate/atropine for the treatment of PI-induced diarrhea. However, in one published trial of six patients previously unresponsive to loperamide therapy, there was no benefit with diphenoxylate/atropine [49]. In addition, as diphenoxylate crosses the bloodbrain barrier at high doses, this potentially leads to dependence and abuse, further discouraging use of this product [73]. As with loperamide, the evidence for use of diphenoxylate/atropine to treat diarrhea in patients with HIV is primarily anecdotal. Despite its long-standing history of availability, this agent should be reserved for patients unresponsive to other options, given its propensity for AEs (including constipation and abuse risk).

\section{Antisecretory Agents}

Octreotide and crofelemer are antisecretory agents with peer-reviewed published data showing their efficacy in treating diarrhea in patients with HIV. These agents inhibit secretory processes within the enterocyte, but the mechanism for their effects, safety profiles, and drug-drug and drug-disease interaction potentials vary greatly.

Octreotide This semisynthetic analogue of somatostatin (a key regulatory protein of the lower gastrointestinal tract that causes slower gastrointestinal transit times) is administered for the symptoms of metastatic carcinoid tumors and vasoactive intestinal peptide secreting adenomas (both resulting in loose, watery diarrhea) [77]. The non-depot formulation of subcutaneously injected octreotide has been administered for symptomatic treatment of diarrhea in patients with HIV; results of published studies have been mostly positive but not limited to noninfectious diarrhea populations [73, 78-82]. Cello et al. [78] evaluated 51 males and females with or without concomitant Mycobacterium, Cryptosporidium or microsporidial infections and refractory diarrhea. Reductions in stool volumes of $50 \%$ or greater over 21 days were reported when patients were treated every $8 \mathrm{~h}$ with incrementally increasing doses of octreotide [78]. In 1996, Beaugerie et al. [79] reported a profound response from a mean \pm SE 
stool frequency at baseline of $5.7 \pm 1.8$ to $1.3 \pm 0.9$ after 1 week of up to thrice daily dosing of octreotide in 3 (23\%) of the study participants. It must be emphasized, however, that octreotide can cause serious, severe hormonal effects (altered balance between insulin, glucagon, and growth hormone, resulting in hypoglycemia and hyperglycemia), along with gastrointestinal disturbances such as gallbladder contractility (resulting in biliary tract abnormalities including sludge); nausea; abdominal discomfort; constipation; and, paradoxically, diarrhea [77]. Equally important is the impact that octreotide, a subcutaneously administered agent, may have on the absorption of orally administered agents, including antiretrovirals. Overall, octreotide is rarely administered in clinical practice for the management of diarrhea in patients with HIV and should only be considered after careful evaluation of risk-benefit.

Crofelemer (SP-303) Crofelemer is an FDAapproved, orally administered, minimally absorbed, botanical drug derived from the stem bark latex of the Croton lechleri tree [83]. Crofelemer is an inhibitor of both the cAMPstimulated CFTR chloride channel and the CaCC; both of these ion channels regulate $\mathrm{Cl}^{-}$ and fluid secretion via intestinal epithelial cells [84] and are proposed as mechanisms of antidiarrheal action for this agent.

Pharmacokinetic data indicate minimal systemic absorption of oral crofelemer, irrespective of concomitant food intake or duration of exposure [41, 83, 85]. Data from in vitro studies have shown that there is no cytochrome P450-mediated metabolism at clinical concentrations of crofelemer, and no clinically relevant drug-drug interactions with nelfinavir, zidovudine, and lamivudine [85]. Although these antiretroviral agents are poor representatives of antiretrovirals commonly used today, the available published literature reflects currently used agents (as described later) without loss of clinical efficacy noted.

The two clinical studies published to date on the use of crofelemer in HIV-associated, noninfectious diarrhea have largely shown positive results using various outcome measures (including somewhat inconsistent and subjective approaches) such as stool weight and volume, patient reported improvement, etc. $[40,41]$.

In the initial phase 2 (randomized, doubleblind, placebo-controlled) study in patients with HIV-associated diarrhea $(N=51 ; 41 / 51$ patients on cART and 39/41 patients on PI), crofelemer treatment $(500 \mathrm{mg}$ every $6 \mathrm{~h}$ for 4 days) resulted in a reduction in mean stool weight compared with placebo on day 4 (451.3 vs $150.7 \mathrm{~g} /$ day; $p=0.14$ ) but only numerically reduced the stool frequency compared with placebo in the same time frame (3.0 vs 2.0 stools/day; $p=0.3$ ) [40].

The second study $(N=374)$ demonstrated that significantly more patients receiving crofelemer $125 \mathrm{mg}$ twice daily obtained a reduction from an average of 2.7 watery stools per day ( $\sim 17$ watery stools per week) to $<2$ watery stools per week for $\geq 2$ of the 4 -week double-blinded, randomized treatment periods compared with placebo $(17.6 \%$ vs. $8.0 \%$, $p=0.01) \quad$ [41]. Other outcome measures favoring crofelemer $125 \mathrm{mg}$ twice daily over placebo were daily watery bowel movements $(-0.96$ vs. $-0.75 ; p=0.04)$ and daily stool consistency score $(-0.43$ vs. $-0.28 ; p=0.02)$. A key determinant of success appears to be patients previously failing to respond to antidiarrheal therapy $(18.1 \%$ vs. $3.5 \%$, $p=0.002)$. Duration of use in the double-blind phase and the 20-week placebo-free extension phase appeared to be consistent with response 
and supports the phase 2 findings of trends toward improvement with exposure [40, 41].

The safety profile for crofelemer was comparable to that of placebo in each dose group. Changes from baseline in HIV clinical parameters (i.e., HIV viral load and CD4+ cell counts) and antiretroviral adherence were minimal, nondetrimental, and comparable between crofelemer and placebo treatment groups during the placebo-controlled phase. The results from these studies suggest that crofelemer should be considered for patients who have failed with over-the-counter loperamide or other antidiarrheal drugs. Importantly, if used, patients must be informed that the benefit is not immediate and improves with continued use. Because crofelemer was not formally studied in combination with loperamide, clinicians should use their judgement to bridge crofelemer onset of effect (anecdotally perceived to be $\sim 4$ weeks by the primary author) with intermittent loperamide use. This hypothesis warrants formal investigation. Also, it is worthwhile noting that if no benefit is seen within 3 months, crofelemer should be discontinued and alternatives considered.

\section{CONCLUSIONS}

Diarrhea in patients with HIV is a significant unmet clinical need that contributes to worsening QoL and complicates the medical management of HIV infection. Because diarrhea incidence is inconsistently reported in clinical trials and package inserts of antiretroviral agents, and because the definitions used to generate rates of diarrhea for patients with HIV receiving cART tend to vary, clinicians are strongly encouraged to question all patients on any cART about their personal experiences with loose, watery stools. Complicating patient management further, the availability of published, peer-reviewed evaluations of treatments for noninfectious diarrhea in patients with HIV is limited. Weighing the entirety of the available information, it appears that if diet modifications, including introduction of psyllium and fiber, fail to resolve a case of confirmed noninfectious diarrhea in patients with HIV, loperamide followed by crofelemer should be considered. Beyond this recommendation, the clinician is encouraged to review the most recent literature available, not relying upon prescribing information. The key to success at each stage of management is for medical professionals to ensure that their patients' incidences of watery stools have been resolved within 1 month of the start of treatment so as to avoid continuation of ineffective therapies and decrease the likelihood of antiretroviral discontinuation.

\section{ACKNOWLEDGMENTS}

Technical editorial and medical writing assistance was provided by Pratibha Hebbar, Ph.D., Synchrony Medical, LLC, West Chester, PA, USA, under the direction of the authors. Sponsorship and article processing charges for this study were funded by Salix Pharmaceuticals, Inc., Raleigh, NC, USA. The authors also wish to thank Kathy Baker, Ph.D., RN, ACNS-BC, FAAN, of Texas Christian University, for her review of the manuscript and comments. All named authors meet the ICMJE criteria for authorship for this manuscript, take responsibility for the integrity of the work as a whole, and have given final approval to the version to be published. 
Conflict of interest. P.G. Clay has received research grants administered by his university from, served as a consultant for, and is a member of the speaker's bureau for Salix Pharmaceuticals, Inc. P. G. Clay has also received grants administered by his university for Entera Health, Inc. R. D. Crutchley has served as a consultant for and is a member of the speaker's bureau for Salix Pharmaceuticals, Inc.

Compliance with ethics. The analysis in this article is based on previously conducted studies and does not involve any new studies of human or animal subjects performed by any of the authors.

Open Access. This article is distributed under the terms of the Creative Commons Attribution Noncommercial License which permits any noncommercial use, distribution, and reproduction in any medium, provided the original author(s) and the source are credited.

\section{REFERENCES}

1. Rockstroh J, Guaraldi G, Deray G. HIV and the body: a review of multidisciplinary management. HIV Med. 2010;11:1-8.

2. Centers for Disease Control and Prevention. HIV surveillance-1981-2008. MMWR Morb Mortal Wkly Rep. 2011;60:689-93.

3. Antiretroviral Therapy Cohort Collaboration. Life expectancy of individuals on combination antiretroviral therapy in high-income countries: a collaborative analysis of 14 cohort studies. Lancet. 2008;372:293-9.

4. Puhan MA, Van Natta ML, Palella FJ, Addessi A, Meinert C. Excess mortality in patients with AIDS in the era of highly active antiretroviral therapy: temporal changes and risk factors. Clin Infect Dis. 2010;51:947-56.

5. Kirk JB, Goetz MB. Human immunodeficiency virus in an aging population, a complication of success. J Am Geriatr Soc. 2009;57:2129-38.
6. MacArthur RD, DuPont HL. Etiology and pharmacologic management of noninfectious diarrhea in HIV-infected individuals in the highly active antiretroviral therapy era. Clin Infect Dis. 2012;55:860-7.

7. Siddiqui U, Bini EJ, Chandarana K, et al. Prevalence and impact of diarrhea on health-related quality of life in HIV-infected patients in the era of highly active antiretroviral therapy. J Clin Gastroenterol. 2007;41:484-90.

8. Tramarin A, Parise N, Campostrini S, et al. Association between diarrhea and quality of life in HIV-infected patients receiving highly active antiretroviral therapy. Qual Life Res. 2004;13:243-50.

9. Prosperi MC, Fabbiani M, Fanti I, et al. Predictors of first-line antiretroviral therapy discontinuation due to drug-related adverse events in HIV-infected patients: a retrospective cohort study. BMC Infect Dis. 2012;12:296.

10. Call SA, Heudebert G, Saag M, Wilcox CM. The changing etiology of chronic diarrhea in HIVinfected patients with CD4 cell counts less than 200 cells $/ \mathrm{mm}^{3}$. Am J Gastroenterol. 2000;95:3142-6.

11. Bode H, Lenzner L, Kraemer OH, et al. The HIV protease inhibitors saquinavir, ritonavir, and nelfinavir induce apoptosis and decrease barrier function in human intestinal epithelial cells. Antivir Ther. 2005;10:645-55.

12. Braga Neto $\mathrm{MB}$, Aguiar $\mathrm{CV}$, Maciel JG, et al. Evaluation of HIV protease and nucleoside reverse transcriptase inhibitors on proliferation, necrosis, apoptosis in intestinal epithelial cells and electrolyte and water transport and epithelial barrier function in mice. BMC Gastroenterol. 2010;10:90.

13. Kotler DP. HIV infection and the gastrointestinal tract. AIDS. 2005;19:107-17.

14. Golder S, Loke YK, Bland M. Meta-analyses of adverse effects data derived from randomised controlled trials as compared to observational studies: methodological overview. PLoS Med. 2011;8:e1001026.

15. Weber R, Ledergerber B, Zbinden R, et al. Enteric infections and diarrhea in human immunodeficiency virus-infected persons: prospective communitybased cohort study. Swiss HIV Cohort Study. Arch Intern Med. 1999;159:1473-80.

16. Knox TA, Spiegelman D, Skinner SC, Gorbach S. Diarrhea and abnormalities of gastrointestinal function in a cohort of men and women with HIV infection. Am J Gastroenterol. 2000;95:3482-9. 
17. Zingmond DS, Kilbourne AM, Justice AC, et al. Differences in symptom expression in older HIVpositive patients: The Veterans Aging Cohort 3 Site Study and HIV Cost and Service Utilization Study experience. J Acquir Immune Defic Syndr. 2003;33:S84-92.

18. daCosta DiBonaventura M, Gupta S, Cho M, Mrus J. The association of HIV/AIDS treatment side effects with health status, work productivity, and resource use. AIDS Care. 2012;24:744-55.

19. Palella FJ Jr, Delaney KM, Moorman AC, et al. Declining morbidity and mortality among patients with advanced human immunodeficiency virus infection. HIV Outpatient Study Investigators. N Engl J Med. 1998;338:853-60.

20. Clotet B, Feinberg J, van LJ, et al. Once-daily dolutegravir versus darunavir plus ritonavir in antiretroviral-naive adults with HIV-1 infection (FLAMINGO): 48 week results from the randomised open-label phase 3b study. Lancet. 2014;383:2222-31.

21. Division of AIDS table for grading the severity of adult and pediatric adverse events. Aug 2009. http://rsc.tech-res.com/Document/safetyandpharm acovigilance/Table_for_Grading_Severity_of_Adult_ Pediatric_Adverse_Events.pdf. Accessed Aug 2013.

22. Antiretroviral drugs used in the treatment of HIV infection. Feb 2013. http://www.fda.gov/ForConsu mers/ByAudience/ForPatientAdvocates/HIVandAID SActivities/ucm118915.htm. Accessed Aug 2013.

23. Hill A, Balkin A. Risk factors for gastrointestinal adverse events in HIV treated and untreated patients. AIDS Rev. 2009;11:30-8.

24. Guest JL, Ruffin C, Tschampa JM, DeSilva KE, Rimland D. Differences in rates of diarrhea in patients with human immunodeficiency virus receiving lopinavir-ritonavir or nelfinavir. Pharmacotherapy. 2004;24:727-35.

25. Molina JM, Andrade-Villanueva J, Echevarria J, et al. Once-daily atazanavir/ritonavir versus twicedaily lopinavir/ritonavir, each in combination with tenofovir and emtricitabine, for management of antiretroviral-naive HIV-1-infected patients: 48 week efficacy and safety results of the CASTLE study. Lancet. 2008;372:646-55.

26. Ortiz R, Dejesus E, Khanlou H, et al. Efficacy and safety of once-daily darunavir/ritonavir versus lopinavir/ritonavir in treatment-naive HIV-1infected patients at week 48. AIDS. 2008;22:1389-97.

27. Eron J Jr, Yeni P, Gathe J Jr, et al. The KLEAN study of fosamprenavir-ritonavir versus lopinavirritonavir, each in combination with abacavirlamivudine, for initial treatment of HIV infection over 48 weeks: a randomised non-inferiority trial. Lancet. 2006;368:476-82.

28. Malan N, Su J, Mancini M, et al. Gastrointestinal tolerability and quality of life in antiretroviral-naive HIV-1-infected patients: data from the CASTLE study. AIDS Care. 2010;22:677-86.

29. Smith KY, Patel P, Fine D, et al. Randomized, double-blind, placebo-matched, multicenter trial of abacavir/lamivudine or tenofovir/emtricitabine with lopinavir/ritonavir for initial HIV treatment. AIDS. 2009;23:1547-56.

30. Gupta R, Ordonez RM, Koenig S. Global impact of antiretroviral therapy-associated diarrhea. AIDS Patient Care STDS. 2012;26:711-3.

31. Riddler SA, Haubrich R, DiRienzo AG, et al. Classsparing regimens for initial treatment of HIV-1 infection. N Engl J Med. 2008;358:2095-106.

32. Katlama C, Haubrich R, Lalezari J, et al. Efficacy and safety of etravirine in treatment-experienced, HIV-1 patients: pooled 48 week analysis of two randomized, controlled trials. AIDS. 2009;23:2289-300.

33. Steigbigel RT, Cooper DA, Kumar PN, et al. Raltegravir with optimized background therapy for resistant HIV-1 infection. $\mathrm{N}$ Engl $\mathrm{J}$ Med. 2008;359:339-54.

34. Dejesus E, Young B, Morales-Ramirez JO, et al. Simplification of antiretroviral therapy to a singletablet regimen consisting of efavirenz, emtricitabine, and tenofovir disoproxil fumarate versus unmodified antiretroviral therapy in virologically suppressed HIV-1-infected patients. J Acquir Immune Defic Syndr. 2009;51:163-74.

35. Dejesus E, Rockstroh JK, Henry K, et al. Coformulated elvitegravir, cobicistat, emtricitabine, and tenofovir disoproxil fumarate versus ritonavirboosted atazanavir plus co-formulated emtricitabine and tenofovir disoproxil fumarate for initial treatment of HIV-1 infection: a randomised, double-blind, phase 3 , non-inferiority trial. Lancet. 2012;379:2429-38.

36. Feasey NA, Healey P, Gordon MA. Review article: the aetiology, investigation and management of diarrhoea in the HIV-positive patient. Aliment Pharmacol Ther. 2011;34:587-603.

37. Rufo PA, Lin PW, Andrade A, et al. Diarrheaassociated HIV-1 APIs potentiate muscarinic activation of $\mathrm{Cl}-$ secretion by $\mathrm{T} 84$ cells via prolongation of cytosolic $\mathrm{Ca}^{2+}$ signaling. Am J Physiol Cell Physiol. 2004;286:C998-1008.

38. Wu X, Sun L, Zha W, et al. HIV protease inhibitors induce endoplasmic reticulum stress and disrupt 
barrier integrity in intestinal epithelial cells. Gastroenterology. 2010;138:197-209.

39. Patel TS, Crutchley RD, Tucker AM, Cottreau J, Garey KW. Crofelemer for the treatment of chronic diarrhea in patients living with HIV/AIDS. HIV AIDS (Auckl). 2013;5:153-62.

40. Holodniy M, Koch J, Mistal M, et al. A double blind, randomized, placebo-controlled phase II study to assess the safety and efficacy of orally administered SP-303 for the symptomatic treatment of diarrhea in patients with AIDS. Am J Gastroenterol. 1999;94:3267-73.

41. MacArthur RD, Hawkins TN, Brown SJ, et al. Efficacy and safety of crofelemer for noninfectious diarrhea in HIV-seropositive individuals (ADVENT trial): a randomized, double-blind, placebo-controlled, twostage study. HIV Clin Trials. 2013;14:261-73.

42. Maresca M, Mahfoud R, Garmy N, Kotler DP, Fantini J, Clayton F. The virotoxin model of HIV1 enteropathy: involvement of GPR15/Bob and galactosylceramide in the cytopathic effects induced by HIV-1 gp120 in the HT-29-D4 intestinal cell line. J Biomed Sci. 2003;10:156-66.

43. Clayton F, Kotler DP, Kuwada SK, et al. Gp120induced Bob/GPR15 activation: a possible cause of human immunodeficiency virus enteropathy. Am J Pathol. 2001;159:1933-9.

44. Brenchley JM, Douek DC. The mucosal barrier and immune activation in HIV pathogenesis. Curr Opin HIV AIDS. 2008;3:356-61.

45. Health Resources and Services Administration HIV/ AIDS Bureau. Guide for HIV/AIDS clinical care. Rockville, MD: U.S. Department of Health and Human Services; Health Resources and Services Administration; HIV/AIDS Bureau; Jan 2011.

46. Sherr L, Lampe F, Norwood S, et al. Successive switching of antiretroviral therapy is associated with high psychological and physical burden. Int J STD AIDS. 2007;18:700-4.

47. Slama L, Li X, Brown T, et al. Increases in duration of first highly active antiretroviral therapy over time (1996-2009) and associated factors in the Multicenter AIDS Cohort Study. J Acquir Immune Defic Syndr. 2014;65:57-64.

48. Mangili A, Murman DH, Zampini AM, Wanke CA. Nutrition and HIV infection: review of weight loss and wasting in the era of highly active antiretroviral therapy from the nutrition for healthy living cohort. Clin Infect Dis. 2006;42:836-42.

49. Sherman DS, Fish DN. Management of protease inhibitor-associated diarrhea. Clin Infect Dis. 2000;30:908-14.
50. Frampton JE. Crofelemer: a review of its use in the management of non-infectious diarrhoea in adult patients with HIV/AIDS on antiretroviral therapy. Drugs. 2013;73:1121-9.

51. Anastasi JK, Capili B, Kim AG, McMahon D, Heitkemper MM. Symptom management of HIVrelated diarrhea by using normal foods: a randomized controlled clinical trial. J Assoc Nurses AIDS Care. 2006;17:47-57.

52. Salminen MK, Tynkkynen S, Rautelin $\mathrm{H}$, et al. The efficacy and safety of probiotic Lactobacillus rhamnosus GG on prolonged, noninfectious diarrhea in HIV patients on antiretroviral therapy: a randomized, placebo-controlled, crossover study. HIV Clin Trials. 2004;5:183-91.

53. Huffman FG, Walgren ME. L-glutamine supplementation improves nelfinavir-associated diarrhea in HIV-infected individuals. HIV Clin Trials. 2003;4:324-9.

54. Conteas CN, Panossian AM, Tran TT, Singh HM. Treatment of HIV-associated diarrhea with curcumin. Dig Dis Sci. 2009;54:2188-91.

55. Anastasi JK, McMahon DJ. Testing strategies to reduce diarrhea in persons with HIV using traditional Chinese medicine: acupuncture and moxibustion. J Assoc Nurses AIDS Care. 2003;14:28-40.

56. Hawkins T. Nelfinavir-associated diarrhea is manageable with nonprescription medications [abstract]. Presented at: The XII International AIDS Conference; Dec 1998; 1998 Jun 30.

57. Vazquez E. Oat bran treats diarrhea. Posit Aware. 1999;10:16.

58. Dylag K, Hubalewska-Mazgaj M, Surmiak M, Szmyd J, Brzozowski T. Probiotics in the mechanism of protection against gut inflammation and therapy of gastrointestinal disorders. Curr Pharm Des. 2014;20:1149-55.

59. Andrews JM, Tan M. Probiotics in luminal gastroenterology: the current state of play. Intern Med J. 2012;42:1287-91.

60. Guinane S. The effectiveness of probiotics for managing diarrhoea in people with HIV infection: a critically appraised topic. HIV Med. 2013;14:187-90.

61. Anukam KC, Osazuwa EO, Osadolor HB, Bruce AW, Reid G. Yogurt containing probiotic Lactobacillus rhamnosus GR-1 and L. reuteri $\mathrm{RC}-14$ helps resolve moderate diarrhea and increases CD4 count in HIV/ AIDS patients. J Clin Gastroenterol. 2008;42:239-43. 
62. Hummelen R, Changalucha J, Butamanya NL, et al. Effect of 25 weeks probiotic supplementation on immune function of HIV patients. Gut Microbes. 2011;2:80-5.

63. Asmuth DM, Ma ZM, Albanese A, et al. Oral serumderived bovine immunoglobulin improves duodenal immune reconstitution and absorption function in patients with HIV enteropathy. AIDS. 2013;27:2207-17.

64. EnteraGam [package insert]. Ankeny, Iowa: Entera Health, Inc.; 2014.

65. Coëffier M, Hecketsweiler B, Hecketsweiler P, Dechelotte P. Effect of glutamine on water and sodium absorption in human jejunum at baseline and during PGE1-induced secretion. J Appl Physiol. 2005;98:2163-8.

66. Dulbecco P, Savarino V. Therapeutic potential of curcumin in digestive diseases. World J Gastroenterol. 2013;19:9256-70.

67. Nwachukwu CE, Okebe JU. Antimotility agents for chronic diarrhoea in people with HIV/AIDS. Cochrane Database Syst Rev. 2008;CD005644.

68. DuPont HL, Ericsson CD, Farthing MJ, et al. Expert review of the evidence base for self-therapy of travelers' diarrhea. J Travel Med. 2009;16:161-71.

69. Kollaritsch H, Paulke-Korinek M, Wiedermann U. Traveler's diarrhea. Infect Dis Clin North Am. 2012;26:691-706.

70. Zwas FR, Cirillo NW, El-Serag HB, Eisen RN. Colonic mucosal abnormalities associated with oral sodium phosphate solution. Gastrointest Endosc. 1996;43:463-6.

71. Ilboudo D, Kadio A, Monny L, Mpele P, NdriYoman T, Vohito MD. Therapeutic effect of activated attapulgite mormoiron in related diarrhea syndrome of acquired immunodeficiency (AIDS). Med d'Afrique Noire. 1997;44:307-12.

72. Mwachari CW, Meier AS, Muyodi J, Gatei W, Waiyaki P, Cohen CR. Chronic diarrhoea in HIV1-infected adults in Nairobi, Kenya: evaluation of risk factors and the WHO treatment algorithm. AIDS. 2003;17:2124-6.

73. Morpeth SC, Thielman NM. Diarrhea in patients with AIDS. Curr Treat Options Gastroenterol. 2006;9:23-37.

74. Imodium A-D and Imodium multi-symptom relief (loperamide and loperamide-simethicone) [package insert]. Fort Washington, PA: McNeil Consumer Healthcare; 2005.

75. Mukwaya G, MacGregor T, Hoelscher D, et al. Interaction of ritonavir-boosted tipranavir with loperamide does not result in loperamideassociated neurologic side effects in healthy volunteers. Antimicrob Agents Chemother. 2005;49:4903-10.

76. Sadeque AJ, Wandel $\mathrm{C}$, He $\mathrm{H}$, Shah S, Wood AJ. Increased drug delivery to the brain by P-glycoprotein inhibition. Clin Pharmacol Ther. 2000;68:231-7.

77. Sandostatin [package insert]. East Hanover, NJ: Novartis Pharmaceuticals Corporation; 2012.

78. Cello JP, Grendell JH, Basuk P, et al. Effect of octreotide on refractory AIDS-associated diarrhea. A prospective, multicenter clinical trial. Ann Intern Med. 1991;115:705-10.

79. Beaugerie L, Baumer $\mathrm{P}$, Chaussade $\mathrm{S}$, et al. Treatment of refractory diarrhoea in AIDS with acetorphan and octreotide: a randomized crossover study. Eur J Gastroenterol Hepatol. 1996;8:485-9.

80. Romeu J, Miro JM, Sirera G, et al. Efficacy of octreotide in the management of chronic diarrhoea in AIDS. AIDS. 1991;5:1495-9.

81. Simon DM, Cello JP, Valenzuela J, et al. Multicenter trial of octreotide in patients with refractory acquired immunodeficiency syndrome-associated diarrhea. Gastroenterology. 1995;108:1753-60.

82. Garcia Compean D, Ramos Jimenez J, Guzman de la Garza F, et al. Octreotide therapy of large-volume refractory AIDS-associated diarrhea: a randomized controlled trial. AIDS. 1994;8:1563-7.

83. Cottreau J, Tucker A, Crutchley R, Garey KW. Crofelemer for the treatment of secretory diarrhea. Expert Rev Gastroenterol Hepatol. 2012;6:17-23.

84. Tradtrantip L, Namkung W, Verkman AS. Crofelemer, an antisecretory antidiarrheal proanthocyanidin oligomer extracted from Croton lechleri, targets two distinct intestinal chloride channels. Mol Pharmacol. 2010;77:69-78.

85. Fulyzaq (crofelemer) delayed-release tablets [package insert]. Raleigh, NC: Salix Pharmaceuticals, Inc.; 2013. 